\title{
Computational analysis to predict false-lumen perfusion and outcome of type B aortic dissection
}

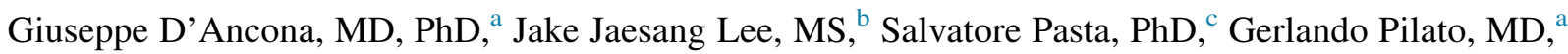 \\ Antonino Rinaudo, MS, ${ }^{\mathrm{c}}$ Fabrizio Follis, MD, ${ }^{\mathrm{a}}$ and Michele Pilato, MD, ${ }^{\mathrm{a}}$ Palermo, Italy, and Pittsburgh, Pa
}

Management of type B aortic dissection is controversial, and the risk of acute or late complications is increased whenever the false lumen (FL) remains patent and perfused. In these patients, computational modeling of baseline computed tomographic images may elucidate complex hemodynamic data, such as the flow patterns in the true lumen (TL) and FL and the intraluminal pressure.

We have attempted to identify potential predictors for acute and late aortic events starting from admission computed tomographic images. Aortic anatomic data and computationally derived variables (FL blood flow and endoluminal pressure) were assessed in patients with type $\mathrm{B}$ aortic dissection and patent FL. A series of imaging variables was derived at each examination (Figure 1).

Three-dimensional aortic geometry was reconstructed from each patient's computed tomographic images and used as input for the computational modeling. ${ }^{2}$ Multiple reentry tears were observed in several patients and thus reconstructed in 3-dimensional aortic models. Follow-up was used to assess differences in hemodynamic predictors and anatomic variables with time. The following hemodynamic variables were evaluated for each simulation: (1) FL pressure index (FL pressure/TL pressure $\times 100 \%)$ and $(2) \mathrm{FL}$ flow rate (FL flow/TL flow $\times 100 \%$ ).

Of 26 patients with a patent and fully perfused FL, 7 $(27 \%)$ had TL collapse develop and $9(35 \%)$ had a thoracic aneurysm develop.

Hemodynamic characteristics of dissected aortas are illustrated for 4 representative patients with streamlines (Figure 2). For all patients, the systolic flow jet was accelerated when passing through the narrowed lumen of the entry tear. Thus the FL was characterized by a slower-moving flow with a recirculating pattern in the descending aorta. This helical pattern was evident for entry tears more distal to the left subclavian artery (compare Figure 2, A, with

\footnotetext{
From the Mediterranean Institute for Transplantation and Advanced Specialized Therapies (ISMETT), ${ }^{\text {a }}$ Palermo, Italy; the University of Pittsburgh Medical Center (UPMC), ${ }^{\mathrm{b}}$ Pittsburgh, Pa; and the Fondazione Ri.MED, ${ }^{\mathrm{c}}$ Palermo, Italy.

Disclosures: Authors have nothing to disclose with regard to commercial support.

Received for publication April 17, 2014; revisions received June 8, 2014; accepted for publication June 16, 2014; available ahead of print Aug 7, 2014.

Address for reprints: Giuseppe D'Ancona, MD, PhD, ISMETT, Via Tricomi 5, 90127 Palermo, Italy (E-mail: rgea@hotmail.com).

J Thorac Cardiovasc Surg 2014;148:1756-8

$0022-5223 / \$ 36.00$

Copyright $($ C 2014 by The American Association for Thoracic Surgery

http://dx.doi.org/10.1016/j.jtcvs.2014.06.065
}

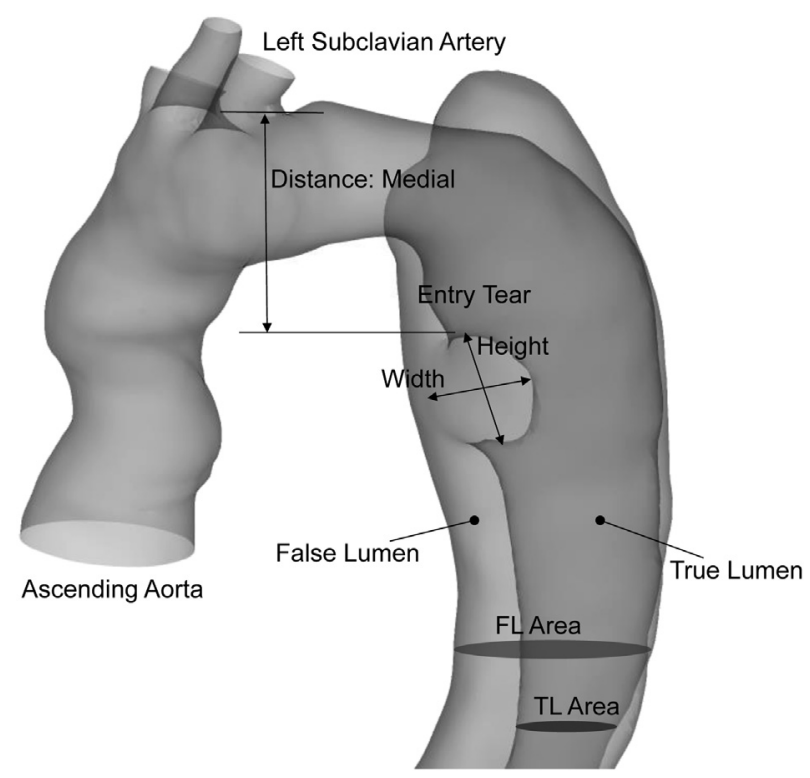

FIGURE 1. Three-dimensional aortic geometry reconstructed from a patient with acute complication showing geometric parameters measured to characterize entry tear and aortic features. For statistical analysis purposes, entry tear location was dichotomized as proximal, medial (as shown by this sketch), or distal from the left subclavian artery; false and true luminal areas were measured perpendicularly to the aortic wall; flow was measured in the false and true luminal areas with Ensight (version 9.0; CEI, Apex, NC). FL, False lumen; $T L$, true lumen.

Figure 2,C). In patients with multiple entry points, the systolic flow entered the FL through both entry and reentry tears simultaneously. In diastole, there was outflow from all tears, suggesting that most of the volume entered and then left the FL from the same tear, rather than going from a proximal entry toward a distal exit. Flow entering the TL was less complicated than that of the FL, with minimal deviance from the inner curvature of the TL wall and an absence of recirculating vortexes.

Multivariate analysis showed that entry tear height was the sole independent determinant of FL flow $(P=.04$; odds ratio, $1.2 ; 95 \%$ confidence interval, 0.02-2.3). Receiver operating characteristic statistics showed that TL area of $148 \mathrm{~mm}^{2}$ (TL diameter of $13.7 \mathrm{~mm}$ ) at admission was the cutoff value to predict acute TL collapse (area under the curve, $0.7 ; P=.03$ ). Moreover, FL area of $1180 \mathrm{~mm}^{2}$ (FL diameter of $38 \mathrm{~mm}$ ) at admission was the cutoff value to predict aneurysmal evolution at follow-up (area under the curve, $0.8 ; P=.01$ ). 

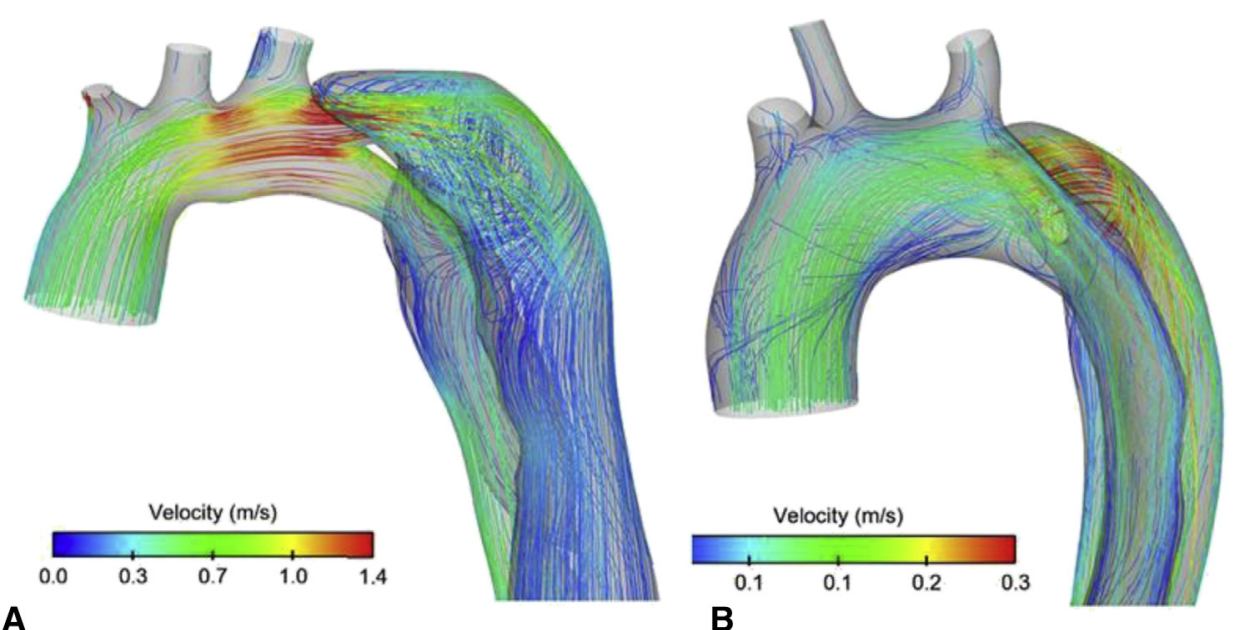

A

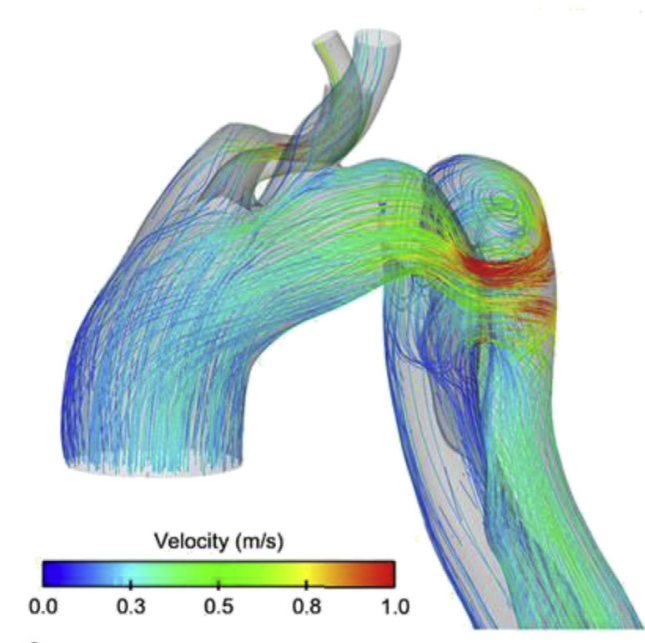

C

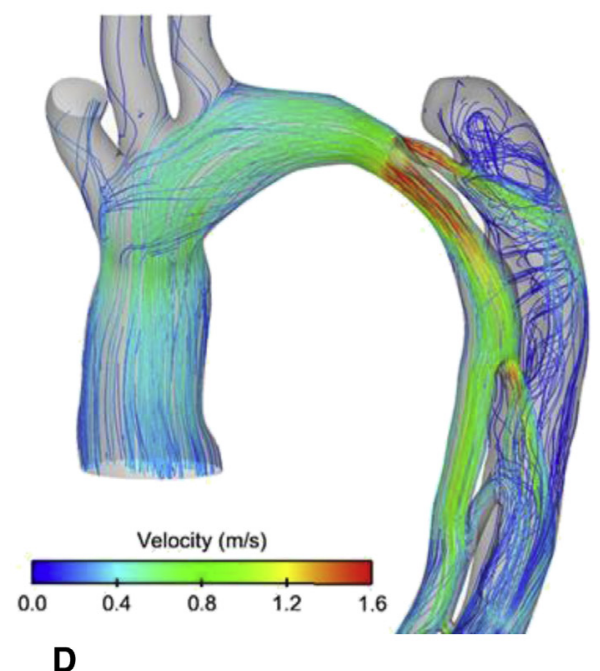

FIGURE 2. Systolic flow velocities derived computationally for a patient with aneurysmal evolution (A), an uncomplicated case (B), a patient with acute complications (C), and a patient with aneurysmal evolution (D).

\section{DISCUSSION}

Hemodynamics after aortic dissection are affected by the aortic anatomy and tear morphology. Evangelista and associates $^{3}$ have previously demonstrated the importance of primary entry tear size and location for predicting follow-up outcome of both type A and B aortic dissections with patent FL. Recently, entry tears located at the concavity of the distal aortic arch were found to be associated with increased risk of acute complications. ${ }^{4}$ Our computational analysis confirms a negative prognostic effect of tear anatomy, which is mainly related to a marked increase in the FL blood flow. Moreover, the altered FL flow illustrated by our computational modeling may lead to progressive, adverse aortic wall remodeling.

Acute evolution of type B aortic dissection remains a big challenge, and at present there are few anatomic predictors of TL acute collapse. ${ }^{5}$ In our analysis, TL size at admission was the strongest indicator of collapse or malperfusion, and this variable should guide close monitoring or preemptive endovascular treatment. In addition, our findings suggest a strong relationship between FL size at first admission and long-term evolution of the aortic anatomy to aneurysm. One can extrapolate from this that a large and highly perfused patent FL mandates long-term monitoring for aneurysmal change.

In conclusion, the proximal entry tear height has a prognostic implication for the blood flow circulating in the patent FL of a type B aortic dissection. Although prediction of specific dissection-related events on the basis of a single morphological parameter or even a combination of parameters is somewhat challenging, information obtained by computational flow analysis could be combined with imaging findings to help in identifying patients at greater risk of dissectionrelated events. With this assumption, when FL perfusion persists, the anatomic variables significantly related to acute or late evolution of type B aortic dissection are TL and FL sizes. 


\section{References}

1. Nienaber CA, Rousseau H, Eggebrecht H, Kische S, Fattori R, Rehders TC, et al. Randomized comparison of strategies for type B aortic dissection: the INvestigation of STEnt Grafts in Aortic Dissection (INSTEAD) trial. Circulation. 2009;120:2519-28. 2. Rinaudo A, D'Ancona G, Lee JJ, Pilato G, Amaducci A, Baglini R, et al. Predicting outcome of aortic dissection with patent false lumen by computational flow analysis. Cardiovasc Eng Technol. 2014;5:176-88.

3. Evangelista A, Salas A, Ribera A, Ferreira-González I, Cuellar H, Pineda V, et al. Long-term outcome of aortic dissection with patent false lumen: predictive role of entry tear size and location. Circulation. 2012;125: 3133-41.

4. Loewe C, Czerny M, Sodeck GH, Ta J, Schoder M, Funovics M, et al. A New Mechanism by Which an Acute Type B Aortic Dissection Is Primarily Complicated, Becomes Complicated, or Remains Uncomplicated. Ann Thorac Surg. 2012;93:1215-22.

5. Tsai TT, Evangelista A, Nienaber CA, Myrmel T, Meinhardt G, Cooper JV, et al. Partial thrombosis of the false lumen in patients with acute type B aortic dissection. N Engl J Med. 2007;357:349-59. 